\title{
Comparative in vitro investigation of the cariogenic potential of bifidobacteria
}

\author{
Remberto Marcelo Argandoña Valdez ${ }^{a}$, Vanessa Rodrigues dos Santos ${ }^{a}$, \\ Karina Sampaio Caiaffa ${ }^{\mathrm{b}}$, Marcelle Danelon ${ }^{\mathrm{a}}$, Rodrigo Alex Arthur ${ }^{\mathrm{c}}$, \\ Thais de Cássia Negrini ${ }^{\mathrm{d}}$, Alberto Carlos Botazzo Delbem ${ }^{\mathrm{a}}$, Cristiane Duque ${ }^{\mathrm{a}, *}$ \\ a UNESP - Univ. Estadual Paulista, Araçatuba Dental School, Department of Pediatric Dentistry and Public Health, Rua José Bonifácio, 1193, 16015050, \\ Araçatuba, SP, Brazil \\ ${ }^{\mathrm{b}}$ UNESP - Univ. Estadual Paulista, Araçatuba Dental School, Department of Restorative Dentistry, Rua José Bonifácio, 1193, 16015050, Araçatuba, SP, Brazil \\ ${ }^{\mathrm{c}}$ UFRGS - Federal University of Rio Grande do Sul, Faculty of Dentistry, Department of Preventive Dentistry, Rua Ramiro Barcelos, 2492, Bairro Santa Cecilia, \\ 90035-003, Porto Alegre, RS, Brazil \\ d UFRGS - Federal University of Rio Grande do Sul, Faculty of Dentistry, Department of Conservative Dentistry, Rua Ramiro Barcelos, 2492, Bairro Santa \\ Cecília, 90035-003, Porto Alegre, RS, Brazil
}

\section{A R T I C L E I N F O}

\section{Article history:}

Received 17 January 2016

Received in revised form 3 June 2016

Accepted 14 July 2016

\section{Keywords:}

Biofilms

Bifidobacterium

Lactobacillus

Streptococcus

Actinomyces

Dental caries

\begin{abstract}
A B S T R A C T
Objective: This study aimed to assess the in vitro cariogenic potential of some Bifidobacterium species in comparison with caries-associated bacteria.

Design: Bifidobacterium lactis, Bifidobacterium longum, Bifidobacterium animalis, Bifidobacterium dentium, Lactobacillus acidophilus, Lactobacillus casei, Actinomyces israelii, Streptococcus sobrinus and Streptococcus mutans were tested for acidogenicity and aciduricity by measuring the $\mathrm{pH}$ of the cultures after growth in glucose and bacterial growth after exposure to acid solutions. Biofilm biomass was determined for each species either alone or associated with S. mutans or S. mutans/S. sobrinus. Enamel hardness was analyzed before and after 7-days biofilm formation using bacterial combinations.

Results: $B$. animalis and B. longum were the most acidogenic and aciduric strains, comparable to cariesassociated bacteria, such as $S$. mutans and L. casei. All species had a significantly increased biofilm when combined either with $S$. mutans or with $S$. mutans/S. sobrinus. The greatest enamel surface loss was produced when B. longum or B. animalis were inoculated with S. mutans, similar to L. casei and S. sobrinus. All strains induced similar enamel demineralization when combined with S. mutans/S. sobrinus, except by B. lactis.

Conclusion: The ability to produce acidic environments and to enhance biofilm formation leading to increased demineralization may mean that Bifidobacterium species, especially B. animalis and B. longum, are potentially cariogenic.
\end{abstract}

(c) 2016 Elsevier Ltd. All rights reserved.

\section{Introduction}

Dental caries is a biofilm-associated disease highly associated with frequent intake of dietary sugars. Fermentation of these sugars by biofilm microorganisms leads to acid production, which disrupts biofilm microbial homeostasis and can causes dissolution of tooth minerals (Marsh, 2003). The bacteria considered the most cariogenic are the mutans streptococci, especially Streptococcus mutans (van Houte, Sansone, Joshipura, \& Kent, 1991; Mattos-

\footnotetext{
* Corresponding author.

E-mail addresses: cristianeduque@yahoo.com.br, cduque@foa.unesp.br (C. Duque).
}

Graner, Correa, Latorre, Peres, \& Mayer, 2001). Other acidogenic and aciduric bacterial species, including Actinomyces spp. and Lactobacillus spp., have been observed in dental caries lesion development (Sansone, van Houte, Joshipura, Kent, \& Margolis, 1993; van Houte, Lopman, \& Kent, 1996). Additionally, Lactobacillus and Bifidobacterium species have been identified as part of the dental biofilm on white spot lesions (Van Ruyven, Lingstrom, van Houte, \& Kent, 2000).

The Bifidobacteriaceae family consists of seven genera: Bifidobacterium, Aeriscardovia, Falcivibrio, Gardnerella, Parascardovia, Scardovia and Alloscardovia, which have been isolated from both animals and humans. These bacteria are anaerobic, gram-positive, rod-shaped, and principally colonize the gastrointestinal tract. Species in the genus Bifidobacterium have recently received 
significant attention in the consumer food industry due to their beneficial roles in human health. They have been shown to have a role in increasing the adaptive immune response, in treating or preventing respiratory and urogenital infections, and in the prevention of allergies and atopic diseases during childhood (Saxelin, Tynkkynen, Mattila-Sandholm, \& de Vos, 2005). Several studies suggest that the consumption of products containing probiotics, such as bifidobacteria, could suppress $S$. mutans levels in saliva (Caglar et al., 2005, 2007, 2008; Cildir et al., 2009). However, recent clinical randomized studies have shown that the administration of probiotics did not affect the mutans streptococci levels or the occurrence of dental caries in children (Taipale, Pienihäkkinen, Salminen, Jokela, \& Söderling, 2012; Taipale, Pienihäkkinen, Alanen, Jokela, \& Söderling, 2013; Nozari, Motamedifar, Seifi, Hatamizargaran, \& Ranjbar, 2015). A confounding issue is that levels of caries-related microorganisms are not always related to the severity of dental caries (Mattos-Graner, Zelante, Line, \& Mayer, 1998), making the beneficial effects of probiotics on the control of caries difficult to elucidate.

Bifidobacterium species have been isolated from dental plaque, saliva and dentinal caries (Modesto, Biavati, \& Mattarelli, 2006; Beighton et al., 2008; Mantzourani et al., 2009). B. dentium, B. longum and $B$. breve were only detected in the oral cavities of children with occlusal caries and not in those of caries-free children (Mantzourani et al., 2009). Those authors suggested that these bacteria might have a role in the progression of occlusal caries lesions. These findings suggest that this family of bacteria may be related to caries development, possibly due to their acidogenicity and acid-tolerance (van Houte, Lopman, \& Kent, 1996; Nakajo, Takahashi, \& Beighton, 2010). Tanner et al. (2011) reported a high prevalence of the Bifidobacterium spp. in addition to Streptococcus in children with advanced dental caries and severe early childhood caries. Thus, this study aimed to determine the cariogenic potential of Bifidobacterium species in comparison with currently recognized caries-associated bacteria. The null hypotheses formulated was that the cariogenic potential of Bifidobacterium species, represented by acidogenicity, aciduricity, and the ability to form biofilms and to induce enamel demineralization, is not different compared with cariogenic potential of caries-associated bacteria.

\section{Materials and methods}

\subsection{Bacterial strains and growth conditions}

Assays were performed using the following Bifidobacterium species: B. animalis (from ACTIVIA ${ }^{\circledR}$ ), B. longum (ATCC 15707), B. lactis (LMG 18905) and B. dentium (ATCC 27678); Lactobacillus species: L. acidophilus (ATCC 4356), L. casei (ATCC 393); Streptococcus species: S. mutans (ATCC 25175 and 3VF2), Streptococcus sobrinus (ATCC 27607); Actinomyces species: A. israelii (ATCC 12102). All ATCC strains were obtained from Oswaldo Cruz Foundation (FIOCRUZ, Rio de Janeiro, RJ, Brazil) or André Tosello Foundation (Campinas, SP, Brazil). B. animalis was isolated from the yogurt ACTIVIA $^{\circledR}$ in Transgalactosylated Olygosaccharides-Propionate agar with supplement lithium-muporicina $(50 \mathrm{mg} / \mathrm{L})$ (TOSMUP agar; Merck Millipore, Darmstadt, Germany). S. mutans 3VF2 is highly acidogenic clinical strain kindly provided by Dr. Renata de Oliveira Mattos-Graner (FOP-UNICAMP) (Mattos-Graner, Napimoga, Fukushima, Duncan, \& Smith, 2004). Reactivation of strains on selective media was done as follow: S. mutans and S. sobrinus on Mitis Salivarius agar with bacitracin $(0.2 \mathrm{U} / \mathrm{mL}$ ) (Difco), L. casei and L. acidophilus on Rogosa agar (Difco); B. animalis, B. longum, B. lactis and $B$. dentium on TOS-MUP agar (Merck) and $A$. israelii on Actinomyces Garrod medium (HiMedia Laboratories, Mumbai,
India). Plates were incubated anaerobically with Anaerocult system (Merck).

\subsection{Acidogenicity tests}

Up to 5 colonies of each species were individually transferred from agar plates to Brain Heart Infusion broth (BHI, Difco) and incubated at $37^{\circ} \mathrm{C}$ for $24 \mathrm{~h}$. Bacterial cultures were diluted 1:20 in BHI and the bacterial growth was monitored by turbidimetry. When the optical density reached 0.5 (approximately $10^{8}-10^{9} \mathrm{CFU} /$ $\mathrm{mL}$ ) at $550 \mathrm{~nm}$, tubes were centrifuged and the pellet was resuspended in a solution of $50 \mathrm{mM} \mathrm{KCl}$ and $1 \mathrm{mM} \mathrm{MgCl}$. The suspension was centrifuged and the pellet was resuspended in $20 \mathrm{mM}$ phosphate buffer and incubated at $37^{\circ} \mathrm{C}$ for $60 \mathrm{~min}$ for starvation. After centrifugation, the pellet was resuspended in a solution of $50 \mathrm{mM} \mathrm{KCl}$ and $1 \mathrm{mM} \mathrm{MgCl}_{2}$, the $\mathrm{pH}$ of the solution was adjusted to 7.0 and glucose was added to a final concentration of $55.5 \mathrm{mM}$. The $\mathrm{pH}$ of the suspension was evaluated immediately and $5,15,30,60,120,180$ and $240 \mathrm{~min}$ after the addition of glucose using a glass electrode (Orion $720 \mathrm{~A}+$; Orion Research Inc.) previously calibrated with $\mathrm{pH}$ standards of $\mathrm{pH} 4.0$ and 7.0. The area under the curve (AUC) was calculated of $\mathrm{pH}$ drop, considering $\mathrm{pH}$ 2.8 as a cut-off point, using UTHSCSA ImageTool software, version 3.0. The acidogenicity was expressed as the AUC $\left(\mathrm{cm}^{2}\right)$. Each analysis was performed in three distinct experiments (Arthur et al., 2011; Belli \& Marquis, 1991).

\subsection{Aciduricity tests}

Similar to acidogenicity tests, $24 \mathrm{~h}$ broth cultures were diluted and grew until optical density at $550 \mathrm{~nm}$ of $0.5 .3 \mathrm{~mL}$ of each culture were individually distributed into tubes, which were centrifuged and resuspended in $0.1 \mathrm{M}$ glycine buffer with a $\mathrm{pH}$ of $7.0, \mathrm{pH} 5.0$ or 2.8. Immediately after resuspension (Time 0 ) and after $60 \mathrm{~min}$ (Time 60 ), $100 \mu \mathrm{L}$ of each sample was serially diluted and plated on BHI agar plates which were incubated for $24 \mathrm{~h}$ at $37^{\circ} \mathrm{C}$. Colony Forming Units $/ \mathrm{mL}(\mathrm{CFU} / \mathrm{mL}$ ) were then counted (Arthur et al., 2011; Duarte et al., 2008).

\subsection{In vitro biofilm formation}

After growing in BHI containing $1 \%$ sucrose and reaching an optical density of 0.5 at $550 \mathrm{~nm}$, aliquots of $200 \mu \mathrm{L}$ of each strain were individually transferred to the wells of 96-well plates (singlespecies). Additionally, equal volumes of each bacterial culture were also combined (at the same optical density) with S. mutans (3VF2) (dual-species) or S. mutans (3VF2)/S. sobrinus (multi-species) to complete a total of $200 \mu \mathrm{L}$ per well. Triplicate of samples were incubated at $37^{\circ} \mathrm{C}$ for $48 \mathrm{~h}$. Wells containing only the culture medium were used as negative controls. After incubation, the plates were washed by immersion in distilled water three times to remove the non-adherent cells. After a brief drying, $150 \mu \mathrm{L}$ of aqueous $1 \%$ crystal violet was added to each well, and the plates were incubated at room temperature for $30 \mathrm{~min}$. The crystal violet solution was then removed and the plates washed again 3 times. The plates were inverted on paper towels and remained for $2 \mathrm{~h}$ at room temperature to dry. The crystal violet dye that stained the biofilm was then solubilized by incubating with $200 \mu \mathrm{L}$ of ethanol per well for $30 \mathrm{~min}$. Then, $100 \mu \mathrm{L}$ of the dye in ethanol was transferred to wells of a new plate, and read at absorbance of $575 \mathrm{~nm}$ in a microplate spectrophotometer (BioTek Instruments. USA) to quantify the biomass of the biofilm (Mattos-Graner, Jin et al., 2001). 


\subsection{In vitro initial caries lesion induction}

This part of the study was approved by the Ethics Committee of Araçatuba Dental School-UNESP (protocol 197/2013). Bovine permanent central incisors were maintained one month in $2 \%$ formaldehyde solution, pH 7.0, for disinfection. Enamel blocks $(4 \times 4 \mathrm{~mm})$ were prepared from the most flat portion of the labial surface of the crown using a precision saw (IsoMet 1000, Buehler, Lake Bluff, IL, USA) with two diamond disks (series 15HC 11-4243Diamond. Buehler) separated by a spacer disk (thickness, $4 \mathrm{~mm}$ ) under refrigeration with distilled water. Dentin was adjusted to obtain parallel surfaces between enamel and dentin $( \pm 2 \mathrm{~mm}$ thick), using sandpaper (CARBIMET, Buehler) under low speed and cooling. Then, the blocks were sequentially polished using 600-, 800 - and 1200-grade water-cooled silicon carbide paper disks (Buehler) with a final polish using a felt disk (Buehler Polishing Cloth 40-7618) moistened with a 1- $\mu \mathrm{m}$ diamond polishing suspension (Extec Corp., Enfield, CT, USA) (Vieira et al., 2005). Blocks with a surface hardness (SH) between 320 and $380 \mathrm{KHN}$ were selected and randomized using Excel program (Microsoft Inc.) into study groups. Twenty enamel blocks were used per group, ten of them or the combination of the bacterial species with Streptococcus mutans (3FV2) (duals-species) and another ten specimens in combination with $S$. mutans and $S$. sobrinus (multispecies). Control groups of $S$. mutans and $S$. mutans/S. sobrinus were also analyzed. The modified protocol of a previous study (Lima, Motisuki, Spolidorio, \& Santos-Pinto, 2005) was used to induce artificial caries lesions. The bovine enamel blocks were completely isolated with a thin layer of nail varnish, except for the external surface (area $=16 \mathrm{~mm}^{2}$ ) and individually placed in modified artificial caries solution (BHI supplemented with $1 \%$ yeast extract, $0.5 \%$ glucose, $1 \%$ sucrose and $2 \%$ of the bacterial culture $-10^{8}$ cells/ $\mathrm{mL}$ ) for 7 days at $37^{\circ} \mathrm{C}$. exchanging the culture medium every $48 \mathrm{~h}$. The blocks were measured for microhardness of the enamel surface before (SH1) and after demineralization (SH2) by means of five impressions alongside the initial five impressions, at a distance of $100 \mu \mathrm{m}$ using a Shimadzu HMV-2000 microhardness tester (Shimadzu Corp., Kyoto. Japan), with a load of $25 \mathrm{~g}$ for $10 \mathrm{~s}$. The percentage of surface hardness loss $(\% \mathrm{SHL}=[[\mathrm{SH} 2-\mathrm{SH} 1] / \mathrm{SH} 1]$ $\times 100$ ) was then calculated.

\subsection{Statistical analysis}

Means (standard deviations) of area under the curve (AUC) of $\mathrm{pH}$ values obtained for bacterial strains during $\mathrm{pH}$ drop (acidogenicity) were calculated and the comparison among bacterial species were tested using ANOVA and Tukey tests. For aciduricity assays, $\mathrm{CFU} / \mathrm{mL}$ counts were transformed to $\log _{10}$ (CFU). The means of counts obtained at pH 7.0 T0 and T60 were calculated and considered as $100 \%$ of cell growth. Counts of viable cells at pH 5.0 and $\mathrm{pH} 2.8$ were also performed and the percentage of viable cells was calculated based on counts at $\mathrm{pH} 7.0$ and data was analyzed using ANOVA and Tukey tests. Absorbance values $(550 \mathrm{~nm})$ obtained after $48 \mathrm{~h}$ of bacterial biofilm formation were compared among the groups of bacteria and between each species combined (dual or multi-species) or single species according to ANOVA and Tukey tests. Percentage of surface hardness loss (SHL) induced by dual-species and multi-species biofilm exposure after 7 days was analyzed using ANOVA and Tukey tests. Virulence factors comparisons among the groups of bacteria (Streptococcus, Lactobacillus and Bifidobacterium) were performed using ANOVA and Tukey (acidogenicity/aciduricity/single-species biofilm) or unpaired $t$-Student tests (dual/multi-species biofilm). Statistical analysis was performed using the program SPSS version 17.1, considering $\mathrm{p}<0.05$ as significant.

\section{Results}

\subsection{Acidogenicity tests}

Fig. 1 shows the results of the AUC for the bacteria tested in this study. Statistically lower values of AUC (high acidogenicity) were found for S. sobrinus, S. mutans (3VF2 and ATCC), L. casei, B. animalis, $B$. longum and $A$. israelii, which were not statistically different from each other. B. lactis, B. dentium and L. acidophilus did not differ from each other but they had the highest values of AUC (low acidogenicity).

\subsection{Aciduricity tests}

Fig. 2 shows means/standard deviations of the percentage of bacterial growth at $\mathrm{pH} 5.0$ and $\mathrm{pH} 2.8$ relative to growth at $\mathrm{pH} 7.0$ (considered 100\%). Analyzing each bacterial strain separately (upper case letters), no difference on percentage of bacterial growth at $\mathrm{pH} 5.0$ between $\mathrm{T} 0$ and $\mathrm{T} 60$ was found for all the tested strains. The percentage of viable cells decreased at $\mathrm{pH} 2.8$ for all bacteria, except for $B$. animalis, B. dentium, L. acidophilus and L. casei, comparing T0 with T60. The percentage of viable cells at $\mathrm{pH}$ 2.8 was statistically lower when compared to $\mathrm{pH} 5.0$ at T0 for all tested bacteria, except for B. longum, $L$. casei and S. mutans ATCC. Additionally, the percentage of viable cells was statistically lower at pH 2.8 after T60 in comparison with pH 5.0 T60 for all strains tested, except for $L$. casei.

Considering each $\mathrm{pH}$ and time separately (lower case letters) at pH 5.0 and T0 the percentage of viable cells of S. mutans (ATCC) was not different compared with $B$. animalis, but it was statistically lower compared with the other strains. At pH 2.8 and T0 the highest percentage of viable cells was observed for B. longum, $L$.

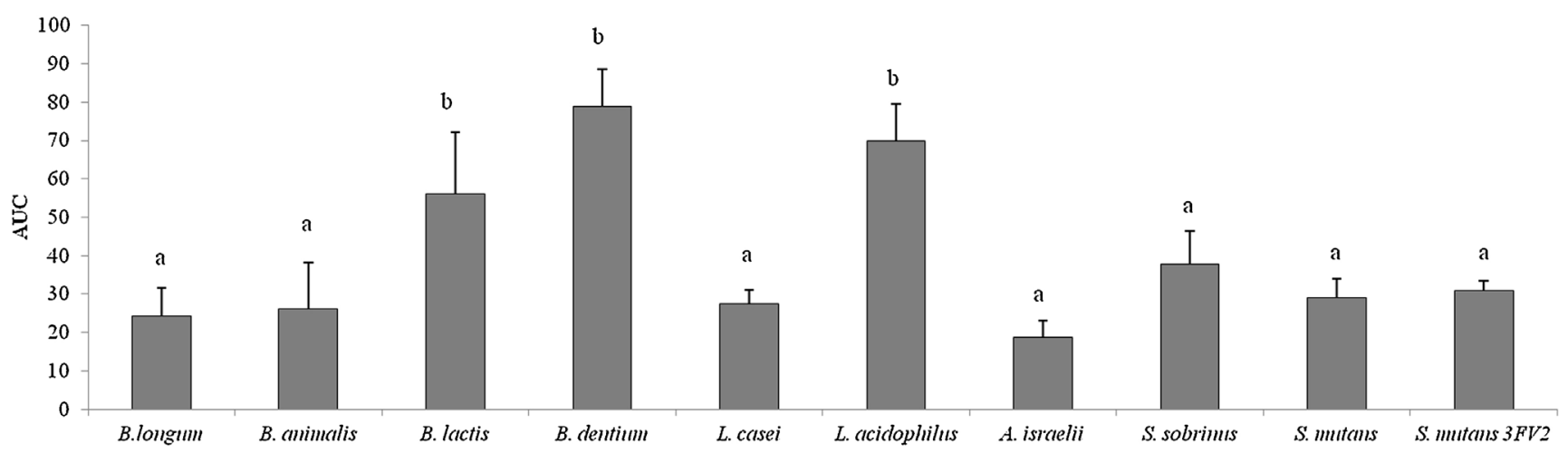

Fig. 1. Acidogenicity (acid production). Means (standard deviations) of area under the curve (AUC) of pH values obtained for bacterial strains during pH drop. ${ }^{A}$ Different lower case letters show statistical difference among bacterial species, according to ANOVA followed by Tukey tests ( $\mathrm{p}<0.05$ ). 


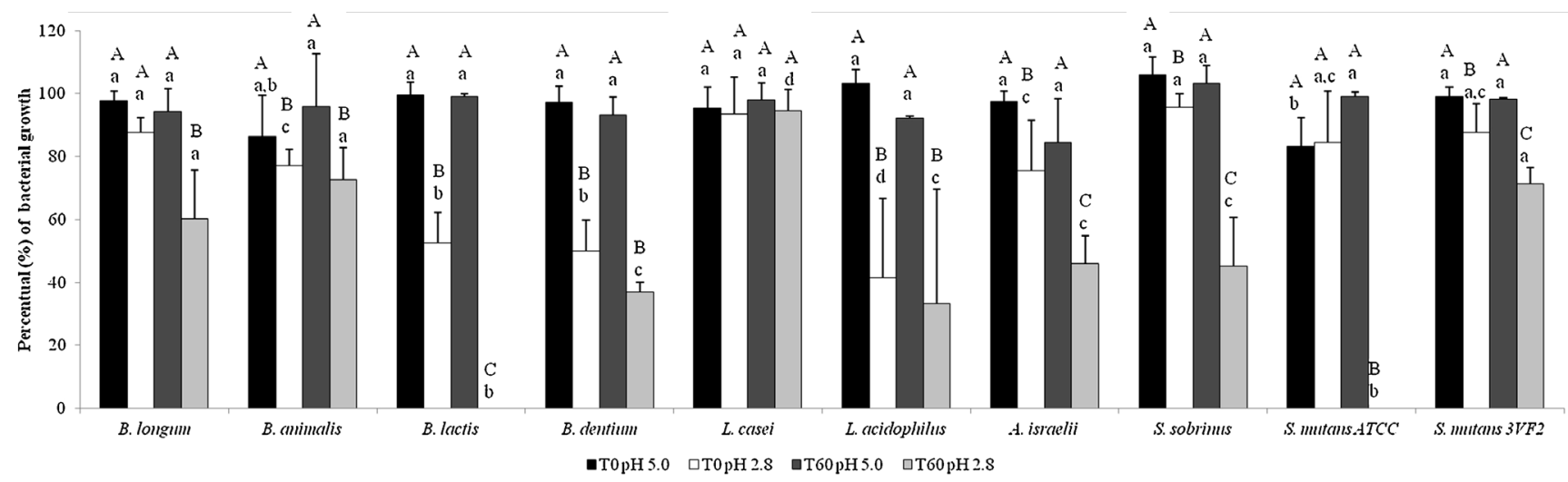

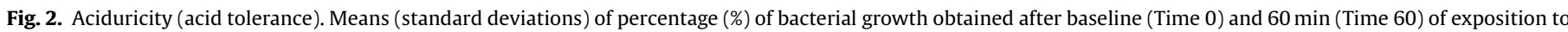
glycine buffer in pH 5.0 or pH. 2.8 (pH 7.0 was considered as 100\%).

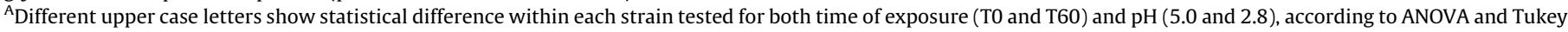
tests $(\mathrm{p}<0.05)$.

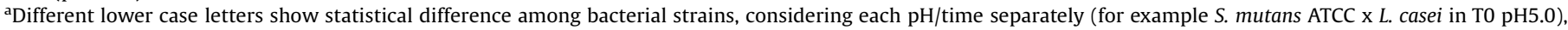
according to ANOVA and Tukey tests $(\mathrm{p}<0.05)$.

casei and S. sobrinus, but without statistical difference from $S$. mutans (ATCC and 3VF2). At pH 5.0 and T60, no difference was found in the percentage of viable cells among all tested strains. At pH 2.8 and T60, L. casei presented the highest aciduricity, followed by $B$. longum and B. animalis, followed by B. dentium, L. acidophilus, A. israelii, S. sobrinus and S. mutans 3VF2, while no growth was observed for B. lactis and S. mutans (ATCC).

\subsection{In vitro biofilm formation}

Fig. 3 shows means/standard deviations of optical density values (Abs $550 \mathrm{~nm}$ ) for the bacterial strains evaluated. For singlespecies biofilms, the ability of $S$. sobrinus to form biofilms was similar to that of $S$. mutans 3VF2, which was higher than biofilms for the other strains. Bifidobacteria and lactobacilli did not form biofilm well. In the dual-species biofilms (associated with $S$. mutans 3VF2), B. animalis followed by L. casei and L. acidophilus had the lowest rates of biofilm formation and $A$. israelii had the highest values, without statistical difference in comparison with $S$. sobrinus and $B$. longum. In the multi-species biofilm, the association of $S$. mutans $3 \mathrm{VF} 2+S$. sobrinus $+B$. dentium presented lower biofilm ability than the combination of $S$. mutans $3 \mathrm{VF} 2+S$. sobrinus and $A$. israelii or $L$. acidophilus or B. longum, but it was not different compared with the other strains combinations. For all the tested strains, the biofilm formation ability was statistically lower as single-species compared with dual- and multi-species biofilms except for $S$. sobrinus single-species compared with $S$. sobrinus dual-species. Multi-species growth of B. longum, B. animalis, L. casei and $L$. acidophilus presented higher biofilm formation ability compared with their growth as dual-species while no difference was found in biofilm formation ability for B. lactis, B. dentium and $A$. israelii between dual- or multi-species growth.

\subsection{In vitro initial caries lesion induction}

Fig. 4 shows surface hardness data (\%SHL) after exposure of enamel specimens to dual and multi-species biofilms. Under dualspecies biofilms, higher demineralization was found in the

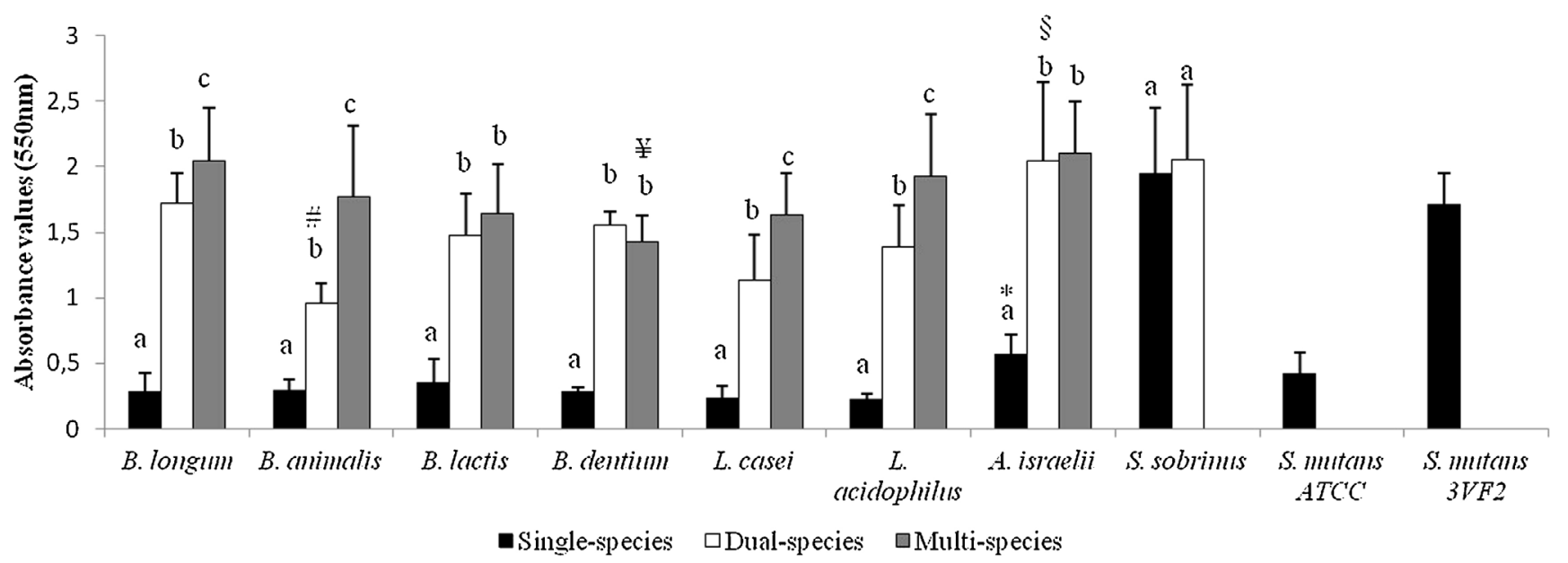

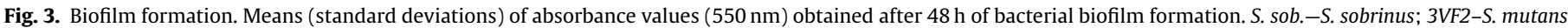
3VF2.

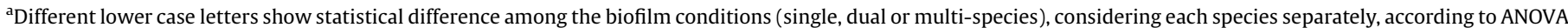
and Tukey tests.

*Single-species biofilm: Statistical difference between A. israelii and other species, according to ANOVA and Tukey tests.

\#Dual-species biofilm: Statistical difference between B. animalis and other species, except by $L$. casei and $L$. acidophilus, according to ANOVA and Tukey tests.

§Dual-species biofilm: Statistical difference between $A$. israelii and other species, except by B. longum, according to ANOVA and Tukey tests.

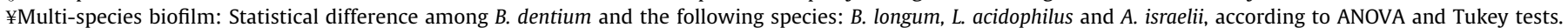




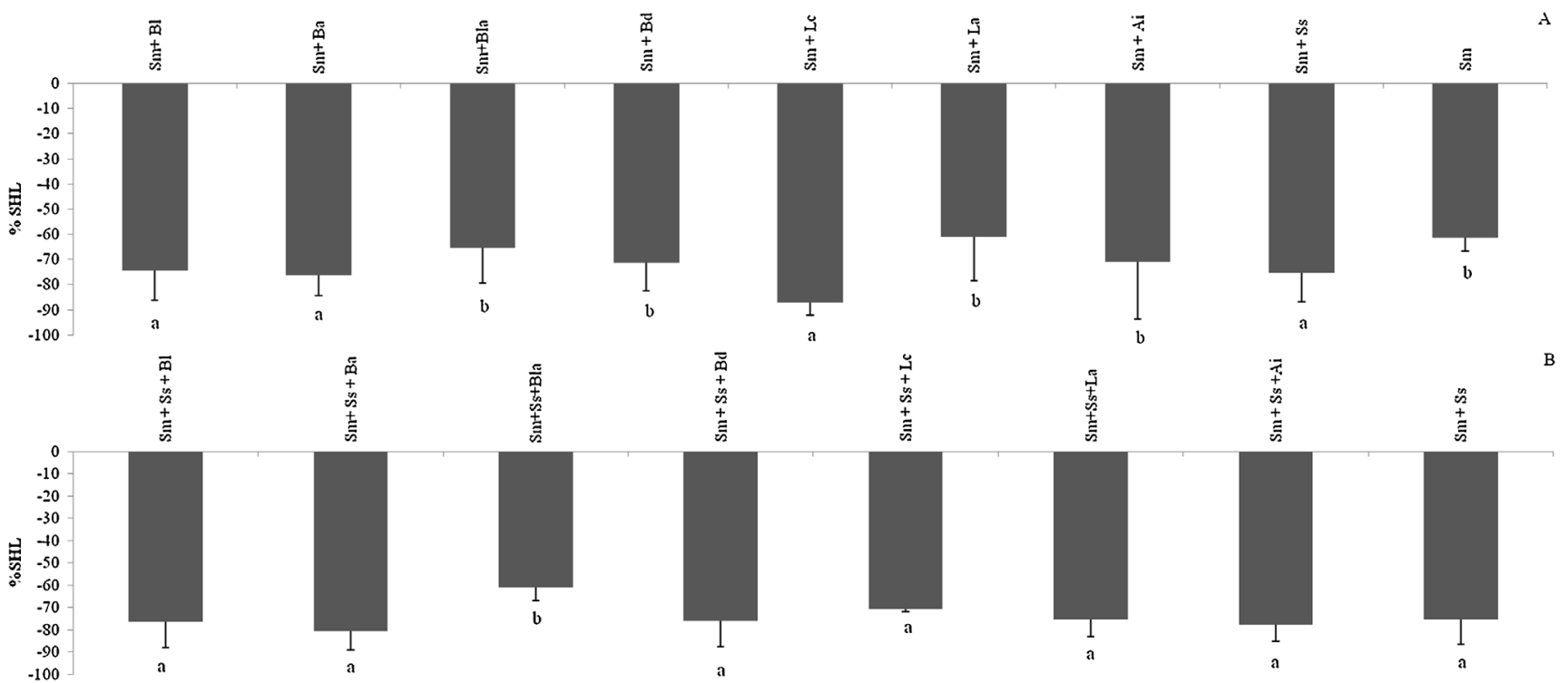

Fig. 4. Enamel demineralization. Percentage of surface hardness loss (\%SHL) after 7 days of (A) dual-species and (B) multi-species biofilm exposure. Sm: S. mutans 3VF2, Ss: S. sobrinus, Bl: B. longum, Ba: B. animalis, Bla: B.lactis, Bd: B. dentium, Lc: L. casei, La: L. acidophilus, Ai: A. israelii ${ }^{a}$ Different letters show statistical difference among the groups, according to ANOVA and Tukey tests.

presence of B. longum. B. animalis, L. casei and S. sobrinus while under multi-species biofilms. B. lactis associated with $S$. mutans 3VF2 and S. sobrinus presented the lowest cariogenic potential compared with the other tested conditions.

\subsection{Comparison of the virulence factors among the groups of microorganisms}

Table 1 summarizes the comparisons among the groups of bacteria in virulence factors evaluated. Lactobacillus and Streptococcus species were more aciduric than Bifidobacterium species. No difference was found among the groups of microorganisms in acidogenicity. Lactobacillus and Bifidobacterium species presented similar biofilm formation and induced similar enamel demineralization either in dual-species or in multi-species biofilms.

\section{Discussion}

Studies have assessed the microbial succession of tooth enamel lesions and noted that various species including Bifidobacterium species, in addition to $S$. mutans, may be involved in the development of early caries lesions (Modesto, Biavati, \& Mattarelli, 2006; Beighton et al., 2008; Mantzourani et al., 2009; Torlakovic et al., 2012). The levels of bifidobacteria, streptococci and lactobacilli have been also compared in caries-free and caries-active individuals. Kaur et al. (2013) found that salivary bifidobacterial levels were significantly correlated $(\mathrm{P}<0.001)$ with salivary levels of mutans streptococci $(r=0.732)$ and lactobacilli $(r=0.625)$. The frequency of detection of mutans streptococci, lactobacilli and bifidobacteria was significantly higher in cariesactive than in the caries-free children, without statistical difference among these bacteria. In adults, salivary levels of bifidobacteria were also significantly related to caries experience and that the salivary levels among mutans streptococci, lactobacilli and bifidobacteria were significantly correlated (Beighton et al., 2010). Specific phenotypic characteristics can influence establishment of species in complex microbial biofilms. The main virulence factors of $S$. mutans that determine their cariogenicity include acidogenicity (production of acids from a variety of fermentable sugars), aciduricity (tolerance to a low $\mathrm{pH}$ ) and ability to form biofilms on the tooth surface, through adhesion and production of extra and intracellular polysaccharides (Lemos, Abranches, \& Burne, 2005).

Data from acidogenicity tests in this current study indicated that, in addition to S. mutans (clinical strain). L. casei, B. animalis and $B$. longum were more acidogenic than the other bacterial

Table 1

Virulence factors comparisons among the groups of microorganisms.

\begin{tabular}{|c|c|c|c|c|}
\hline & & Streptococcus & Lactobacillus & Bifidobacterium \\
\hline $\begin{array}{l}\text { Acidogenicity } \\
\text { (AUC) }\end{array}$ & & $32.58(2.13)^{\mathrm{A}}$ & $52.06(6.74)^{\mathrm{A}}$ & $46.29(7.41)^{\mathrm{A}}$ \\
\hline Aciduricity (\% bacterial growth) & & $62.33(7.35)^{\mathrm{A}}$ & $64.07(5.95)^{\mathrm{A}}$ & $40.30(5.42)^{\mathrm{B}}$ \\
\hline $\begin{array}{l}\text { Biofilm formation } \\
\qquad(\text { Abs values }-550 \mathrm{~nm})\end{array}$ & $\begin{array}{l}\text { Mono-species } \\
\text { Dual-species } \\
\text { Multi-species }\end{array}$ & $\begin{array}{l}1.35(0.03)^{\mathrm{A}} \\
- \\
-\end{array}$ & $\begin{array}{l}0.31(0.03)^{\mathrm{B}} \\
1.22(0.05)^{\mathrm{A}} \\
1.78(0.07)^{\mathrm{A}}\end{array}$ & $\begin{array}{l}0.30(0.02)^{\mathrm{B}} \\
1.41(0.06)^{\mathrm{A}} \\
1.70(0.06)^{\mathrm{A}}\end{array}$ \\
\hline $\begin{array}{l}\text { Enamel demineralization } \\
\quad(\% \mathrm{SHL})\end{array}$ & $\begin{array}{l}\text { Dual-species } \\
\text { Multi-species }\end{array}$ & - & $\begin{array}{l}-69.91(4.44)^{\mathrm{A}} \\
-72.47(1.77)^{\mathrm{A}}\end{array}$ & $\begin{array}{l}-71.75(2.35)^{\mathrm{A}} \\
-73.61(2.40)^{\mathrm{A}}\end{array}$ \\
\hline
\end{tabular}

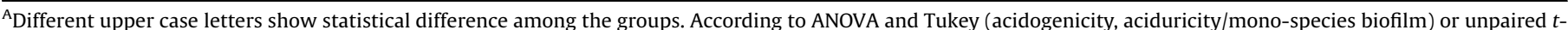
Student tests (dual/multi-species biofilm).

Means (Standard Error of Means). 
strains analyzed. S. sobrinus and $A$. israelii were among the most acidogenic strains, but had intermediate acid tolerance. The $\mathrm{pH}$ drops caused by probiotics, including B. longum, B. lactis, L. casei and $L$. acidophilus, from the degradation of sucrose and other sugars were evaluated by Haukioja, Loimaranta, \& Tenovuo (2008). The authors found that all of the strains produced acids from glucose with a reduction of $\mathrm{pH}$ comparable to that of $S$. mutans, similar to the present results. Those authors also found that all lactobacilli and bifidobacteria tested, except for L. rhamnosus GG and $B$. lactis $\mathrm{Bb} 12$, caused a significant decrease in $\mathrm{pH}$ in the presence of lactose.

The current study showed that the growth of all strains, including species of bifidobacteria, was not affected by pH 5.0 after $60 \mathrm{~min}$ of acid exposure. The same was not observed at $\mathrm{pH} 2.8$. Among bifidobacteria, $B$. longum and $B$. animalis were the most acid-resistant strains and $B$. dentium was the least acid-resistant strain, at $\mathrm{pH}$ 2.8. These results are in agreement with those obtained by Nakajo, Takahashi, \& Beighton (2010) who evaluated the effects of acidification on the survival of $B$. dentium and $B$. longum in comparison with $S$. mutans, $S$. sanguinis and $L$. paracasei. High levels of survival in acidic environments were observed for bifidobacteria, which were comparable to S. mutans, demonstrating how these species could co-exist with Streptococcus mutans in acidic carious lesions. $B$. longum was the most resistant bacteria at pH 5.0 when compared to the others strains, while $L$. paracasei was the most resistant at $\mathrm{pH}$ 4.0. In contrast, $B$. dentium was less resistant to acids among all tested bacteria.

Bacterial aciduricity is maintained by a mechanism in the cell membrane called proton-translocating ATPase (F1F0-ATPase). This mechanism controls the entry of $\mathrm{H}+$ ions and maintaining a more basic cytoplasmic $\mathrm{pH}$ when compared to the extracellular medium. Furthermore, the F-ATPase system has dual role in the acid tolerance of cells and eliminating protons in certain circumstances it generates ATP (energy) for the growth and persistence of bacterial species (Lemos \& Burne, 2008). Studies have reported that the F-ATPase activity in Bifidobacterium animalis and Bifidobacterium animalis subsp lactis increases with environmental acidification (Gibson \& Wang, 1994; Sánchez, de los Reyes-Gavilán, \& Margolles, 2006). Matsumoto, Ohishi, \& Benno (2004) evaluated the aciduricity and F-ATPase activity of B. lactis (2 strains), $B$. animalis (4 strains), B. bifidum (4 strains). B. breve, B. infantis, $B$. catenulatum, $B$. longum, $B$. pseudocatenulatum and $B$. adolescentis and demonstrated that $B$. lactis and $B$. animalis were able to survive in an acidic environment (they were stable at $\mathrm{pH} 3-5$ for $3 \mathrm{~h}$ ). They reported that this ability was related to the species and not the strain and was associated with increased F-ATPase activity. In contrast with the current study findings, Matsumoto et al. (2004) reported that $B$. longum was not acid tolerant, with reduced growth by about 3 -fold at $\log 10$ after exposure to $\mathrm{pH} 5$ for $3 \mathrm{~h}$. Other ATPindependent mechanisms have been studied in $S$. mutans, $S$. gordonii, S. salivarius and L. casei (Fozo \& Quivey, 2004; Fozo, Kajfasz, \& Quivey, 2004). The membrane composition may be altered in the presence of acids, increasing the levels of long-chain monounsaturated fatty acids, allowing the species to become more resistant to acids. The cell membrane of $B$. animalis contains large amounts of these fatty acids (Ruiz, Sánchez, Ruas-Madiedo, de Los Reyes-Gavilán, \& Margolles, 2007), suggesting that these bifidobacteria may also display this type of acid tolerance.

In this present study, bifidobacteria and lactobacilli had lower ability to form biofilm as single-species compared with dual- and multi-species biofilms. Multi-species growth of B. longum, $B$. animalis, $L$. casei and $L$. acidophilus presented higher biofilm formation ability compared with their growth as dual-species. Previous studies had demonstrated that some bifidobacteria ( $B$. breve, $B$. longum, $B$. lactis, $B$. adolescentis, $B$. infantis) exhibited low adhesion to hydroxyapatite discs coated or not coated with saliva
(Haukioja et al., 2006; Nagaoka et al., 2008). This ability was improved when the species had co-adhered with primary colonizers, such as A. naeslundii, V. parvula, and F. nucleatum.

Due their lower capacity to adhere to tooth and to form biofilm by themselves, enamel demineralization was induced only by lactobacilli and bifidobacteria combined with $S$. mutans or with $S$. mutans and $S$. sobrinus. The dual-species biofilms of $S$. mutans with $B$. longum, $L$. casei, $B$. animalis or $S$. sobrinus and the multi-species biofilms of $B$. animalis and $A$. israelii with $S$. mutans and S. sobrinus caused the highest loss of surface hardness. Different combinations of $S$. mutans with $L$. casei and $L$. acidophilus were recently tested to observe the induction of non-cavitated enamel lesions in human teeth (De Campos et al., 2015). Their results showed that dual combination of $S$. mutans and $L$. casei or triple combination of $S$. mutans, L. casei and L. acidophilus promoted the highest loss in enamel surface hardness. The depth of lesions was analyzed using polarized light microscopy and erosive lesions developed in enamel after 20 days of cariogenic challenge. The results of the present study are consistent with those results, showing that the combination of $L$. casei and $S$. mutans caused a greater enamel demineralization compared to other species tested. Studies evaluating the impact of the biofilm formed by Bifidobacterium species and $S$. mutans on demineralization of dental enamel have not yet been found, making comparisons with the present results difficult. It could be concluded that the ability to produce acidic environments and to enhance biofilm formation leading to increased enamel demineralization may mean that Bifidobacterium species, especially $B$. animalis and B. longum, are potentially cariogenic.

\section{Conflict of interests}

The authors declare that there are no conflicts of interest.

\section{Ethical approval}

The experimental procedures described here were approved by the Ethics Committee of Araçatuba Dental School - UNESP (protocol 197/2013).

\section{Acknowledgements}

This study was supported by grants (\#2012/19235-5) and scholarship for VRS (\#2014/02072-1) from the São Paulo Research Foundation (FAPESP, São Paulo, Brazil) and scholarship for RMAV (\#141702/2012-5) by the National Board of Scientific and Technological Development (CNPq, Brazil). The authors would like to thank Renata de Oliveira Mattos-Graner (FOP-UNICAMP, Brazil) and Anne C. R. Tanner and Christine A. Kressirer (Forsyth Institute, Cambridge, USA) for providing bacterial strains and for critical review of this manuscript.

\section{References}

Arthur, R. A., Cury, A. A., Mattos-Graner, R. O., Rosalen, P. L., Vale, G. C., Paes Leme, A F., et al. (2011). Genotypic and phenotypic analysis of S. mutans isolated from dental biofilms formed in vivo under high cariogenic conditions. Brazilian Dental Journal, 22, 267-274.

Beighton, D., Gilbert, S. C., Clark, D., Mantzourani, M., Al-Haboubi, M., Ali, F., et al. (2008). Isolation and identification of Bifidobacteriaceae from human saliva. Applied and Environmental Microbiology, 74, 6457-6460.

Beighton, D., Al-Haboubi, M., Mantzourani, M., et al. (2010). Oral Bifidobacteria: Caries-associated bacteria in older adults. Journal of Dental Research, 89, 970-974.

Belli, W. A., \& Marquis, R. E. (1991). Adaptation of Streptococcus mutans and Enterococcus hirae to acid stress in continuous culture. Applied and Environmental Microbiology, 57, 1134-1138.

Caglar, E., Sandalli, N., Twetman, S., Kavaloglu, S., Ergeneli, S., \& Selvi, S. (2005). Effect of youghurt with Bifidobacterium DN-173 010 on salivary mutans 
streptococci and lactobacilli in young adults. Acta Odontologica Scandinavica, 63, 317-320.

Caglar, E., Kavaloglu, S. C., Kuscu, O. O., Sandalli, N., Holgerson, P. L., \& Twetman, S. (2007). Effect of chewing gums containing xylitol or probiotic bacteria on salivary mutans streptococci and lactobacilli. Clinical Oral Investigations, 11, 425-429.

Caglar, E., Kuscu, O. O., Selvi Kuvvetli, S., Kavaloglu Cildir, S., Sandalli, N., \& Twetman, S. (2008). Short-term effect of ice-cream containing Bifidobacterium lactis Bb-12 on the number of salivary mutans streptococci and lactobacilli. Acta Odontologica Scandinavica, 66, 154-158.

Cildir, S. K., Germec, D., Sandalli, N., Ozdemir, F. I., Arun, T., Twetman, S., et al. (2009). Reduction of salivary mutans streptococci in orthodontic patients during daily consumption of yoghurt containing probiotic bacteria. European Journal of Orthodontics, 31, 407-4011.

De Campos, P. H., Sanabe, M. E., Rodrigues, J. A., Duarte, D. A., Santos, M. T., Guaré, R. O., et al. (2015). Different bacterial models for in vitro induction of noncavitated enamel caries-like lesions: Microhardness and polarized light miscroscopy analyses. Microscopy Research and Technique, 78, 444-451.

Duarte, S., Klein, M. I., Aires, C. P., Cury, J. A., Bowen, W. H., \& Koo, H. (2008). Influences of starch and sucrose on Streptococcus mutans biofilms. Oral Microbiology and Immunology, 23, 206-212.

Fozo, E. M., \& Quivey, R. G. Jr. (2004). Shifts in the membrane fatty acid profile of Streptococcus mutans enhance survival in acidic environments. Applied and Environmental Microbiology, 70, 929-936.

Fozo, E. M., Kajfasz, J. K., \& Quivey, R. G. Jr. (2004). Low-pH induced membrane fatty acid alterations in oral bacteria. FEMS Microbiology Letters, 238, 291-295.

Gibson, G. R., \& Wang, X. (1994). Regulatory effects of bifidobacteria on the growth of other colonic bacteria. Journal of Applied Bacteriology, 77, 412-420.

Haukioja, A., Yli-Knuuttila, H., Loimaranta, V., Kari, K., Ouwehand, A. C., Meurman, J. H., et al. (2006). Oral adhesion and survival of probiotic and other lactobacilli and bifidobacteria in vitro. Oral Microbiology and Immunology, 21, 326-332.

Haukioja, A., Loimaranta, V., \& Tenovuo, J. (2008). Probiotic bacteria affect the composition of salivary pellicle and streptococcal adhesion in vitro. Oral Microbiology and Immunology, 23, 336-343.

Kaur, R., Gilbert, S. C., Sheehy, E. C., \& Beighton, D. (2013). Salivary levels of Bifidobacteria in caries-free and caries-active children. International Journal of Paediatric Dentistry, 23, 32-38.

Lemos, J. A., \& Burne, R. A. (2008). A model of efficiency: Stress tolerance by Streptococcus mutans. Microbiology, 154(Pt. 11), 3247-3255.

Lemos, J. A., Abranches, J., \& Burne, R. A. (2005). Responses of cariogenic streptococci to environmental stresses. Current Issues in Molecular Biology, 7, 95-107.

Lima, L. M., Motisuki, C., Spolidorio, D. M., \& Santos-Pinto, L. (2005). In vitro evaluation of probiotics microorganisms adhesion to an artificial caries model. European Journal of Clinical Nutrition, 59, 884-886.

Mantzourani, M., Gilbert, S. C., Sulong, H. N., Sheehy, E. C., Tank, S., Fenlon, M., et al. (2009). The isolation of bifidobacteria from occlusal carious lesions in children and adults. Caries Research, 43, 308-313.

Marsh, P. D. (2003). Are dental diseases examples of ecological catastrophes? Microbiology, 149, 279-294.

Matsumoto, M., Ohishi, H., \& Benno, Y. (2004). H+-ATPase activity in Bifidobacterium with special reference to acid tolerance. International Journal of Food Microbiology, 93, 109-113.

Mattos-Graner, R. O., Zelante, F., Line, R. C., \& Mayer, M. P. (1998). Association between caries prevalence and clinical: microbiological and dietary variables in 1.0-2.5-year-old Brazilian children. Caries Research, 32, 319-323.

Mattos-Graner, R. O., Napimoga, M. H., Fukushima, K., Duncan, M. J., \& Smith, D. J. (2004). Comparative analysis of Gtf isozyme production and diversity in isolates of Streptococcus mutans with different biofilm growth phenotypes. Journal of Clinical Microbiology, 42, 4586-4592.

Mattos-Graner, R. O., Correa, M. S. N. P., Latorre, M. R. O., Peres, R. C. R., \& Mayer, M. P. A. (2001). Mutans streptococci oral colonization in 12-30-month-old Brazilian children over a one year follow-up period. Journal of Public Health Dentistry, 61, $161-167$.

Mattos-Graner, R. O., Jin, S., King, W. F., Chen, T., Smith, D. J., \& Duncan, M. J. (2001). Cloning of the Streptococcus mutans gene encoding glucan binding protein B and analysis of genetic diversity and protein production in clinical isolates. Infection and Immunity, 69, 6931-6941.

Modesto, M., Biavati, B., \& Mattarelli, P. (2006). Occurrence of the family Bifidobacteriaceae in human dental caries and plaque. Caries Research, 40, 271-276.

Nagaoka, S., Hojo, K., Murata, S., Mori, T., Ohshima, T., \& Maeda, N. (2008). Interactions between salivary Bifidobacterium adolescentis and other oral bacteria: In vitro coaggregation and coadhesion assays. FEMS Microbiology Letters, 281, 183-189.

Nakajo, K., Takahashi, N., \& Beighton, D. (2010). Resistance to acidic environments of caries-associated bacteria: Bifidobacterium dentium and Bifidobacterium longum. Caries Research, 44, 431-437.

Nozari, A., Motamedifar, M., Seifi, N., Hatamizargaran, Z., \& Ranjbar, M. A. (2015). The effect of Iranian customary used probiotic yogurt on the children's salivary cariogenic Microflora. Journal of Dentistry (Shiraz), 16, 81-86.

Ruiz, L., Sánchez, B., Ruas-Madiedo, P., de Los Reyes-Gavilán, C. G., \& Margolles, A. (2007). Cell envelope changes in Bifidobacterium animalis ssp. lactis as a response to bile. FEMS Microbiology Letters, 274, 316-322.

Sánchez, B., de los Reyes-Gavilán, C. G., \& Margolles, A. (2006). The F1F0-ATPase of Bifidobacterium animalis is involved in bile tolerance. Environmental Microbiology, 8, 1825-1833.

Sansone, C., van Houte, J., Joshipura, K., Kent, R., \& Margolis, H. C. (1993). The association of mutans streptococci and non-mutans streptococci capable of acidogenesis at a low $\mathrm{pH}$ with dental caries on enamel and root surfaces. Journal of Dental Research, 72, 508-516.

Saxelin, M., Tynkkynen, S., Mattila-Sandholm, T., \& de Vos, W. M. (2005). Probiotic and other functional microbes: From markets to mechanisms. Current Opinion in Biotechnology, 16, 204-211.

Taipale, T., Pienihäkkinen, K., Salminen, S., Jokela, J., \& Söderling, E. (2012) Bifidobacterium animalis subsp. lactis BB-12 administration in early childhood: A randomized clinical trial of effects on oral colonization by mutans streptococci and the probiotic. Caries Research, 46, 69-77.

Taipale, T., Pienihäkkinen, K., Alanen, P., Jokela, J., \& Söderling, E. (2013). Administration of Bifidobacterium animalis subsp. lactis BB-12 in early childhood: A post-trial effect on caries occurrence at four years of age. Caries Research, 47, 364-372.

Tanner, A. C., Kent, R. L. Jr., Holgerson, P. L., Hughes, C. V., Loo, C. Y., Kanasi, E., et al. (2011). Microbiota of severe early childhood caries before and after therapy. Journal of Dental Research, 90, 1298-1305.

Torlakovic, L., Klepac-Ceraj, V., Ogaard, B., Cotton, S. L., Paster, B. J., \& Olsen, I. (2012). Microbial community succession on developing lesions on human enamel. Journal of Oral Microbiology, 4, . http://dx.doi.org/10.3402/jom.v4i0.16125.

Vieira, A. E. M., Delbem, A. C. B., Sassaki, K. T., Rodrigues, E., Cury, J. A., \& Cunha, R. F. (2005). Fluoride dose response in $\mathrm{pH}$-cycling models using bovine enamel. Caries Research, 39, 514-520.

van Houte, J., Sansone, C., Joshipura, K., \& Kent, R. (1991). In vitro acidogenic potential and mutans streptococci of human smooth-surface plaque associated with initial caries lesions and sound enamel. Journal of Dental Research, 7, 1497-1502.

van Houte, J., Lopman, J., \& Kent, R. (1996). The final pH of bacteria comprising the predominant flora on sound and carious human root and enamel surfaces. Journal of Dental Research, 75, 1008-1014.

van Ruyven, F. O., Lingstrom, P., van Houte, J., \& Kent, R. (2000). Relationship among mutans streptococci: Low-pH bacteria. and iodophilic polysaccharideproducing bacteria in dental plaque and early enamel caries in humans. Journal of Dental Research, 79, 778-784. 